\title{
ESSAY
}

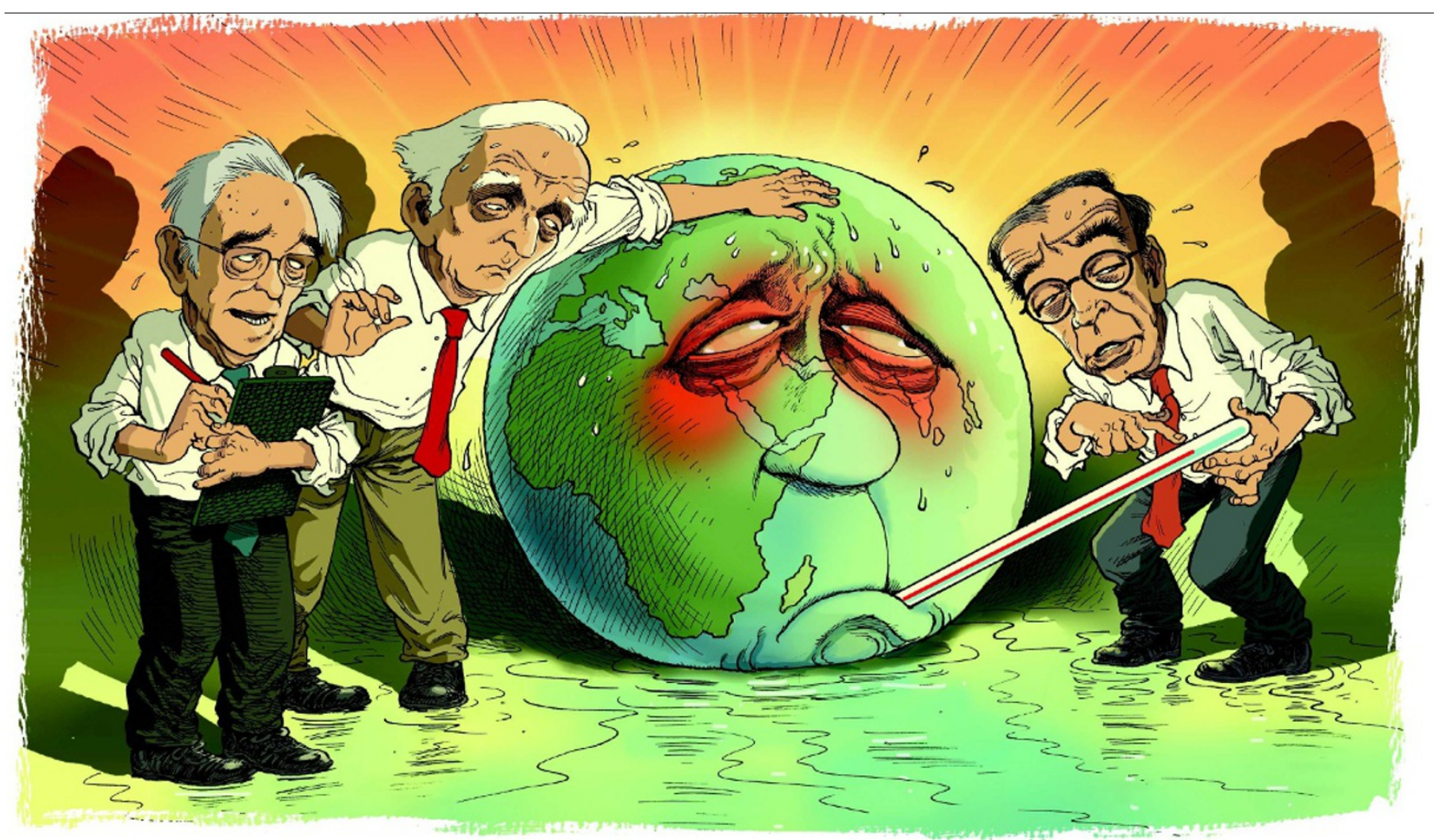

\section{Madrid 1995: Diagnosing climate change}

\section{John Houghton chaired the tense IPCC meeting without which there would be no Kyoto}

Protocol. Here he recalls how science won the day.

Climate change is increasingly recognized as one of the most serious threats to humankind. But it was not always so. That the majority of world leaders are now convinced of this peril, even though its full effects will not be felt for decades, is due largely to the work of one organization: the Intergovernmental Panel on Climate Change (IPCC).

The IPCC draws together hundreds of the world's top scientists to study and assess the effects and consequences of human activity on the climate. As an intergovernmental organization under the United Nations, its five-yearly assessments must be accepted by governments, and the IPCC recommendations are then taken as the basis for policy. Crucially, the content of the final text is determined solely by the science, based on the research literature.

As first chair, and later co-chair, of the IPCC's working group on the physical basis of climate change from 1988 to 2002 , I was present at all the meetings associated with the first three IPCC Reports - in 1990, 1995 and 2001. One meeting was particularly challenging, contentious and ultimately satisfying: this was to agree the IPCC's second assessment report, and was held in Madrid over three days at the end of November 1995.

The mid-1990s were a crucial time in the study of climate change. The science had moved on since the first IPCC assessment in 1990 and there was growing evidence for the impact of human activity. Climate change had stirred worldwide interest, and groups whose interests might be affected by any policies derived from the report were keen to have their say. We knew that the process of agreeing a final text for the 1995 assessment was going to be more controversial than before.

\section{Climate wars}

The Madrid meeting was attended by 177 government delegates from 96 countries, 28 scientists representing the authors of the 11 chapters of the IPCC report, and 14 representatives of non-governmental organizations (NGOs). Some had strong environmentalist agendas. Others represented business and industry. One industry NGO, the Global Climate Coalition (now "deactivated"), was among the most active in challenging the IPCC's conclusions on climate change. Backed by powerful parts of the US and international energy industry, the coalition had begun to take part in the IPCC's activities by attending meetings, and writing and reviewing papers. Its representatives worked closely with the official delegates from Saudi Arabia, Kuwait and other oil- and gas-exporting states.

In preparation for the meeting, a small group containing the leading scientist authors had prepared a shortened version of the main assessment report - a Summary for Policymakers. The aim of the meeting would be to shape this summary text into a form that all those present (government representatives and IPCC scientists) could agree on.

The meeting opened under the co-chairmanship of myself and Luiz Gylvan Meira Filho, former head of Brazil's space agency. We began by discussing the content and conclusions of the 11 chapters, and our deliberations did not seem out of the ordinary compared with previous meetings.

By the second day, as we got to grips with the Summary for Policymakers, things were moving much more slowly than expected. A small core of delegates, led by the representatives of Saudi Arabia and Kuwait, wanted to weaken the 
statements about the extent of climate change and put greater emphasis on the uncertainties associated with the causes of climate change.

Inevitably, the most heated discussions focused on how to summarize Chapter 8 of the full report, which addressed the extent to which human-induced global warming had been observed in the climate record. Before the meeting, several governments, in particular the United States, had pointed out that the summary at the beginning of Chapter 8 was not entirely consistent with the conclusions at the end of the chapter, and that some statements had not been crafted with sufficient care. When the plenary session began, many other countries echoed these views and asked for revisions, which the meeting accepted.

It was during this debate that the delegates from Kuwait and Saudi Arabia asked for words or phrases that emphasized uncertainty regarding human-induced climate change to be inserted in the Summary for Policymakers. Two sentences in the summary were particularly sensitive. The first concerned patterns of temperature change that scientists were observing across the globe. In its draft form it read:

More convincing evidence for the attribution of a human effect on climate is emerging from pattern based studies, in which the modelled climate response to combined forcing by greenhouse gases and anthropogenic sulphate aerosols is compared with observed geographical, seasonal and vertical patterns of atmospheric temperature change."

Saudi Arabia and Kuwait requested that the first three words be altered to "Some preliminary evidence", because the draft version of Chapter 8 had used the phrase "preliminary evidence" in association with pattern-based studies. For an hour and a half, the meeting debated the appropriateness of "preliminary" instead of "convincing". All other delegates who spoke argued that the evidence in Chapter 8 did not warrant the description "preliminary", and a sentence was finally drafted that began "More convincing recent evidence...". The meeting agreed that a footnote should be added to the final text explaining that Saudi Arabia and Kuwait dissented from the majority view. This footnote was later withdrawn at the countries' own request.

The second sentence to spark disagreement read:

The balance of evidence suggests human influence on global climate.

In 1990, the IPCC's report had concluded that unequivocal detection of anthropogenic climate change "would not be likely for a decade or more". So again Saudi Arabia swam against the tide, arguing that "balance of evidence" was far too strong. The debate over this sentence lasted well over an hour and various alternatives were proposed: "The balance/weight/preponderance of evidence points to/suggests/indicates an appreciable/detectable/measurable/discernible human influence on the global climate."

The sentence finally agreed on was: "The balance of evidence suggests a discernible human influence on global climate." The word that most excited the meeting was "discernible": it was suggested at a very late stage by the UK delegate as a replacement for "appreciable", and was spontaneously applauded.

It was a tough meeting but no one lost their cool, and the undoubted tension was punctuated by occasional humour. I joked with Mohamed Al-Sabban, the head of Saudi Arabia's delegation, that if I were a member of his delegation I would advise strongly against a particular proposal he was making, as being against his country's interests. He promptly made me an honorary citizen of Saudi Arabia, later referring a few times to my 'honorary citizenship.

It all finished at 20 minutes past midnight, 10 minutes before the conference centre was due to close, and to my relief no unsatisfactory compromises had been made. When I read through what we had agreed, I realized that the powerful discipline of science had created a final result that was better in its clarity, accuracy and presentation than the draft text with which we had begun.

\section{Integrity attack}

But that is not how our critics interpreted things. The following year, when the full, revised IPCC assessment was published, the Global Climate Coalition accused the IPCC of dishonesty and fraud. On 12 June 1996, the Wall Street Journal published an op-ed by Frederick Seitz, a former president of the US National Academy of Sciences, that accused the IPCC authors of altering Chapter 8 of the 1995 report after it had been formally approved. "I have never witnessed a more disturbing corruption of the peer-review process than the events that led to this IPCC report," Seitz claimed, quoting differences between the draft chapter that had been available to the Madrid meeting and the final published version.

The late Bert Bolin (the IPCC chairman at the time), Gylvan Meira Filho and I wrote to the Wall Street Journal explaining that there was no question of 'corruption' of any kind and that the authors had been working completely within IPCC rules. The newspaper printed a much-shortened version of our reply on 25 June but continued to run articles repeating Seitz's accusations. In addition, for some months afterwards the Global Climate Coalition waged a relentless campaign in the United States against Ben Santer, the lead author of Chapter $8-$ to the extent that the American Meteorological Society felt compelled to publish an open letter praising him and supporting the IPCC.

Happily, the IPCC came through the process with its integrity intact. In 2001 and 2007 it released two further reports that substantially confirmed the conclusions of the first two reports, adding much more detail about the likely impacts of climate change and disclosing significant evidence about positive feedbacks amplifying global warming that had barely been recognized in 1995 .

I am often asked to explain how it is possible for so many scientists from different countries and cultures to come to a consensus about a subject as complex and uncertain as climate change. My reply is that science, with its emphasis on robust data, repeatability, balance, accuracy and integrity and its reliance on argument and debate to reach a conclusion, provides an ideal process for such an endeavour.

Furthermore, the consensus does not, of course, apply to everything: the IPCC has always been at pains to distinguish between what we know with reasonable certainty and those areas in which much is unclear.

Perhaps the most important outcome of the IPCC process has been the creation of a large research community, well informed about all aspects of climate change, which can provide sound information to reduce the confusion bound to arise from such a complex nexus of science and society. The involvement of this community in all aspects of the assessment process has meant that the IPCC's published reports are more punchy and more precise than they would otherwise have been.

In Madrid in 1995, the IPCC scientific assessment process, based on the findings of the latest research, was sorely tested. Had the science not come through unscathed, the integrity of the panel would have been seriously questioned, and governments would have faltered on taking urgent action on climate change, such as the signing in 1997 of the Kyoto Protocol. John Houghton chaired the IPCC meeting in Madrid from 27 to 29 November 1995. He was chairman of the UK Royal Commission on Environmental Pollution from 1992 to 1996 and is currently president of the John Ray Initiative. e-mail: john.houghton@jri.org.uk

For more Meetings that Changed the World, see www/nature.com/nature/focus/meetings. 